\title{
MEDIDAS DE BIOSEGURIDAD QUE APLICA EL PROFESIONAL DE ENFERMERÍA Y SU RELACIÓN CON LA EXPOSICIÓN AL RIESGO LABORAL EN EL HOSPITAL SANTA MARÍA DEL SOCORRO, AÑO 2013 - 2014.
}

Bio-safety measures applying professional nursing and its relation to exposure to working on risk hospital Santa Maria del Socorro of Ica, year 2013-2014.

Wendy Jurado Lengua ${ }^{1, a}$, Silvia Solís Junchaya ${ }^{2, a}$, Carolina Soria Quispe ${ }^{3, a}$

${ }^{1}$ Facultad de Enfermería Universidad Nacional San Luis Gonzaga de Ica, Perú. ${ }^{2}$ Puesto de Salud El Palto - Pachacutec, Ica, Perú.

${ }^{3}$ Puesto de Salud Buena Fe, Nazca - Ica, Perú, ${ }^{a}$ Licenciado(a) en Enfermería.

\section{RESUMEN}

Objetivo: Determinar las Medidas de Bioseguridad que aplica el profesional de enfermería y su relación con la Exposición al Riesgo Laboral en el Hospital Santa María de Socorro, año 20132014. Material y Método: Se realizó un estudio no experimental, descriptivo correlacional. La muestra fue de 57 profesionales de enfermería de diferentes servicios y estrategias de dicho hospital, cuya relación se midió con un instrumento validado por juicio de expertos, el cual consistió de 3 partes: Datos generales, Medidas de bioseguridad y Exposición al riesgo laboral. Resultados: En cuanto a las Barreras de Protección que aplica el profesional de enfermería, encontrándose que guarda una relación inversa baja con la Exposición al riesgo biológico, una relación inversa mínima con la exposición al riesgo físico y una relación directa moderada significativa con la exposición al riesgo químico. Se encontró que existe aplicación de Barreras físicas a menudo 42,11\%, aplicación de Barreras Biológicas, respecto a la Vacuna de Hepatitis B con tres dosis 7,02\% y la Vacuna Toxoide tetánico con tres dosis en un 7,02\%, así mismo existe medidas de precaución estándar respecto al lavado de Manos Siempre en un $97,74 \%$ y respecto a la disponibilidad de desechos Siempre en un $71,93 \%$. Respecto a la exposición del riesgo laboral el 73,68\% afirma haber estado expuesto al riesgo, siendo un pinchazo en el $59,65 \%$ el que pudo haber causado el accidente, así mismo se resalta que hubo un promedio de 12,56 puntos afirma que a menudo existe un riesgo físico y 19,05 puntos afirma que algunas veces existe un riesgo químico. Conclusiones: Existe relación directa Moderada Significativa entre las barreras de protección que aplica el Profesional de Enfermería y la Exposición al Riesgo Químico, relación inversa baja pero no significativa entre las Barreras de Protección que aplica el Profesional de Enfermería y la Exposición al Riesgo biológico y relación inversa mínima pero no significativa entre las Barreras de Protección que aplica el Profesional de Enfermería y la Exposición al Riesgo físico

Palabras Claves: Bioseguridad, Riesgo, Exposición, Barrera, Relación

\section{SUMMARY}

Objective: To determine the Biosecurity Measures applying professional nursing and its relationship with the Labour Exposure Risk in Hospital Santa Maria Socorro, year 2013 - 2014 Material and Methods: A non-experimental, descriptive correlational study was conducted. The sample consisted of 57 nurses from different services and strategies that hospital, whose relationship was measured with a validated instrument expert judgment, which consisted of 3 parts: General information, Biosecurity and labor risk exposure. Results: Concerning the Protection Barriers applying nurse, being keeping a low inverse relationship with biohazard exposure, minimal inverse relationship to exposure to physical risk and a significant moderate direct relation to exposure to chemical risk. Application found that there is often physical $42,11 \%$, application of 
biological barriers, with respect to Hepatitis B Vaccine three doses 7,02\% and the tetanus toxoid vaccine in three doses Barriers 7,02\% and there same standard precautions about washing hands always in $97,74 \%$ and compared to the availability of waste always a $71,93 \%$. Regarding occupational risk exposure the $73.68 \%$ reported having been exposed to risk, being a prick $59,65 \%$ which may have caused the accident, also highlights that there was an average of 12,56 points says there is often a physical hazard and 19,05 points says that sometimes there is a chemical hazard. Conclusions: Moderate Significant direct relationship between guardrails applying Professional Nursing and Chemical Risk Exposure, low but significant inverse relationship between the barriers applying Protection Professional Nursing and Biological Exposure Risk and relationship low but significant inverse Protection Barriers between applying Professional Nursing and Physical Hazard Exposure

Keywords: Biosafety, Risk, Exposure, Barrera, Relation

\section{INTRODUCCIÓN}

La bioseguridad tuvo sus inicios en la guerra de Crimea, desarrollada entre21854 -1856. Durante este conflicto bélico, el 21 de octubre de 1854, Florence Nightingale, fue enviada a la península de Crimea, por el secretario de guerra Sídney Hebert, para que junto a enfermeras voluntarias limpiaran y reformaran el hospital, logrando disminuir de esta manera, la tasa de mortalidad del $40 \%$ al $2 \%$ (1).

Según la Organización Internacional del Trabajo (OIT), en el 2011 informa que alrededor de 337 millones de personas son víctimas de accidentes y enfermedades laborales cada año (2). Así mismo la OMS indica que los incidentes laborales más frecuentes son los accidentes que ocurren con el personal de salud, un tercio de todas las lesiones se presentan en el personal de enfermería debido al uso inadecuado de las medidas de bioseguridad (3).

Los antecedentes internacionales en Venezuela concluyen que en las medidas de bioseguridad no son observadas por un porcentaje importante de la población estudiada evidenciándose que existe una importante incidencia de accidentes laborales por punciones percutáneas y cortaduras. En nuestro país el estudio realizado en Lima por Cárdenas, se llegó a la conclusión que las enfermeras tienen un nivel de conocimiento bajo sobre medidas de bioseguridad y la mayoría de las enfermeras no cumplen con las prácticas de las medidas de bioseguridad durante la atención de los pacientes (4). En Ica, (2008-2011) un estudio realizado por Uribe C, concluyó que el Riesgo laboral está asociado a los factores psicosociales del personal asistencial y administrativo del Hospital Regional de Ica (5).

La bioseguridad, como disciplina nace durante la década del 70, La bioseguridad es el conjunto de medidas preventivas que tienen como objetivo proteger la salud y la seguridad del personal, de los pacientes y de la comunidad; frente a diferentes riesgos producidos por agentes biológicos, físicos, químicos y mecánicos (6).

Este estudio se realiza por iniciativa e interés de las investigadoras que durante las prácticas hospitalarias en dicha institución observaron que las enfermeras de los diferentes servicios, estuvieron en mayor exposición al manejo de fluidos biológicos y/o riesgo a enfermedades ocupacionales debido a los procedimientos que realizan y las condiciones laborales.

Con esta investigación se busca que los resultados sean de utilidad para futuros estudiantes en esta línea de investigación y promover una conducta reflexiva en la prevención logrando un ambiente de trabajo 
ordenado, seguro y conduzca simultáneamente a mejorar la calidad de atención. Para la realización de esta tesis se determinó como objetivo general: Determinar las Medidas de Bioseguridad que aplica el profesional de enfermería y su relación con la Exposición al Riesgo Laboral en el Hospital Santa María de Socorro, año 2013 - 2014 y como objetivos específicos: Determinar la relación entre las medidas de bioseguridad de las barreras de protección que aplica el profesional de enfermería con la exposición al riesgo biológico en el Hospital Santa María del socorro año 2013-2014, Identificar la relación entre las medidas de bioseguridad de las barreras de protección que aplica el profesional de enfermería con la exposición al riesgo físico en el Hospital Santa María del socorro año 2013-2014 y Determinar la relación entre las medidas de bioseguridad de las barreras de protección que aplica el profesional de enfermería con la exposición al riesgo químico en el Hospital Santa María del socorro año 2013-2014.

Planteándose como Hipótesis General: Existe relación entre las Medidas de Bioseguridad que aplica el profesional de enfermería con la Exposición al Riesgo Laboral en el Hospital Santa María de Socorro, año 2013 - 2014

Planteándose el objetivo: determinar la relación que existe entre las variables para conocer qué porcentaje de la muestra en estudio que aplica las medidas de bioseguridad están expuestas al riesgo laboral y haciéndole llegar las conclusiones a las autoridades para que se establezca estrategias que disminuyan el riesgo laboral en el campo clínico y mejorar la calidad de atención, así mismo que sirva de marco de referencia para futuros trabajos de investigación.

\section{MATERIAL Y MÉTODOS}

El estudio utilizó el método descriptivo correlacional de tipo no experimental ejecutándose en el Hospital Santa María del
Socorro que se encuentra ubicada en la ciudad de Ica calle castrovirreyna № 759 ICA-PERÚ, con una población de 70 enfermeros (as) con una muestra constituida de 57 profesionales de enfermería seleccionados de diferentes servicios del hospital.

Se excluyeron a los profesionales de enfermería que laboran menos de 6 meses, a los que se encontraron de vacaciones y de licencia, los que laboraban en el área de administración y a los que no quisieron formar parte de nuestra investigación en el Hospital Santa María Del Socorro.

Se hizo uso de un cuestionario que consto de 3 partes: primera parte: datos generales segunda parte: con preguntas para medir las medidas de bioseguridad, tercera parte: estuvo conformado por preguntas para medir la exposición al riesgo laboral en el cual consta de 51 reactivos estructurados, cerrados, dicotómicas de opción múltiple y de escala likert, utilizándose una escala de alternativas cuyas puntuaciones fueron: nunca (1) casi nunca (2) algunas veces (3) a menudo (4) siempre (5). En la recolección de datos se utilizó como técnica el cuestionario elaborado por las autoras, previamente validado por los juicios de expertos y reestructurado con preguntas de opción múltiple y dicotómicas (si, no) cerradas y de escala likert, para evaluar la confiabilidad del instrumento se utilizó una prueba piloto de 20 $\%$ de la muestra, los que permitieron determinar las características finales del instrumento. Para la confiabilidad del instrumento se aplicó el Alfa de Cronbach.

\section{RESULTADOS}

En cuanto a las Barreras de Protección que aplica el profesional de enfermería se ha encontrado que guarda una relación inversa baja con la Exposición al riesgo biológico, una relación inversa mínima con la exposición al riesgo físico y una relación directa moderada significativa con la exposición al riesgo químico. (Tabla 1) 
También se ha encontrado que existe aplicación de Barreras físicas a menudo 42,11\%, aplicación de Barreras Biológicas, respecto a la Vacuna de Hepatitis $B$ con tres dosis $7,02 \%$ y la Vacuna Toxoide tetánico con tres dosis en un $7,02 \%$, así mismo existe medidas de precaución estándar respecto al lavado de Manos Siempre en un 97,74\% y respecto a la disponibilidad de desechos Siempre en un $71,93 \%$. En cuanto a la exposición del riesgo laboral el 73,68\% afirma haber estado expuesto al riesgo, siendo un pinchazo en el $59,65 \%$ el que pudo haber causado el accidente, así mismo se resalta que hubo un promedio de 12,56 puntos que afirma que a menudo existe un riesgo físico y 19,05 puntos que afirma que algunas veces existe un riesgo químico. (Tabla 2).

\section{Tabla 1 INTERPRETACIÓN DE LA TABLA DE CORRELACIONES ENTRE LAS DIMENSIONES}

\begin{tabular}{|c|c|c|c|}
\hline Correlación & $\begin{array}{l}\text { Barreras de Protección } \\
\text { X1 }\end{array}$ & $\begin{array}{c}\text { Precauciones } \\
\text { Estándar en Lavado } \\
\text { de Manos X2 }\end{array}$ & $\begin{array}{c}\text { Precauciones Estándar en } \\
\text { Disponibilidad de desechos } \\
\text { X3 }\end{array}$ \\
\hline $\begin{array}{c}\text { Exposición al } \\
\text { Riesgo Biológico } \\
\text { Y1 }\end{array}$ & $\begin{array}{c}\text { Rho Spearman } \\
-0,244 \\
\text { Sig=0,067 } \\
\text { Correlación inversa } \\
\text { baja }\end{array}$ & $\begin{array}{c}\text { Rho Spearman } \\
-0,020 \\
\text { Sig=0,882 } \\
\text { Correlación inversa } \\
\text { mínima }\end{array}$ & $\begin{array}{c}\text { Rho Spearman } \\
-0,261^{\star} \\
\text { Sig }=0,050 \\
\text { Correlación inversa baja } \\
\text { significativa }\end{array}$ \\
\hline $\begin{array}{l}\text { Exposición al } \\
\text { Riesgo Físico } \\
\text { Y2 }\end{array}$ & $\begin{array}{c}\text { Rho Spearman } \\
-0,062 \\
\text { Sig=0,652 } \\
\text { Correlación inversa } \\
\text { mínima }\end{array}$ & $\begin{array}{c}\text { Rho Spearman } \\
-0,187 \\
\text { Sig=0,167 } \\
\text { Correlación inversa } \\
\text { mínima }\end{array}$ & $\begin{array}{c}\text { Rho Spearman } \\
-0,003 \\
\text { Sig=0,982 } \\
\text { Correlación inversa mínima }\end{array}$ \\
\hline $\begin{array}{c}\text { Exposición al } \\
\text { Riesgo Químico } \\
\text { Y3 }\end{array}$ & $\begin{array}{c}\text { Rho Spearman } \\
0,442 \\
\text { Sig=0,001 } \\
\text { Correlación directa } \\
\text { moderada significativa }\end{array}$ & $\begin{array}{c}\text { Rho Spearman } \\
0,085 \\
\text { Sig=0,532 } \\
\text { Correlación directa } \\
\text { mínima }\end{array}$ & $\begin{array}{c}\text { Rho Spearman } \\
0,291^{\star} \\
\text { Sig=0,029 } \\
\text { Correlación directa baja } \\
\text { significativa }\end{array}$ \\
\hline
\end{tabular}

TABLA 2 ELEMENTOS EVALUADOS EN LA INVESTIGACIÓN.

\begin{tabular}{|c|c|c|}
\hline ELEMENTOS EVALUADOS & $\mathrm{N}=57$ & $\%$ \\
\hline \multicolumn{3}{|l|}{ MEDIDAS DE BIOSEGURIDAD } \\
\hline \multicolumn{3}{|l|}{${ }^{\star}$ Barreras Físicas } \\
\hline Siempre <64-80] & 19 & 33,33 \\
\hline A menudo $<48-64]$ & 24 & 42,11 \\
\hline Algunas Veces <32-48] & 11 & 19,30 \\
\hline Casi Nunca <16-32] & 3 & 5,26 \\
\hline Nunca $[1-16]$ & 0 & 0 \\
\hline \multicolumn{3}{|l|}{ *Barreras Biológicas } \\
\hline 3 Dosis completa de vacuna Toxoide Tetánico & 4 & 7,02 \\
\hline 3 Dosis completas de Vacuna Hepatitis B & 4 & 7,02 \\
\hline Dosis Única de Vacuna Influenza & 42 & 73,68 \\
\hline Dosis Única de Vacuna BCG & 51 & 89,47 \\
\hline \multicolumn{3}{|l|}{ *Precauciones Estándar } \\
\hline \multicolumn{3}{|l|}{ Lavado de Manos } \\
\hline Siempre<48-60] & 54 & 94,74 \\
\hline A menudo<36-48] & 3 & 5,26 \\
\hline Algunas Veces<24-36] & 0 & 0 \\
\hline Casi Nunca<12-24] & 0 & 0 \\
\hline Nunca[1-12] & 0 & 0 \\
\hline \multicolumn{3}{|l|}{ Disponibilidad de Desechos } \\
\hline Siempre <16-20] & 41 & 71,93 \\
\hline A menudo <12-16] & 13 & 22,81 \\
\hline Algunas Veces <8-12] & 3 & 5,26 \\
\hline Casi Nunca <4-8] & 0 & 0 \\
\hline Nunca [1-4] & 0 & 0 \\
\hline
\end{tabular}




\begin{tabular}{|c|c|c|}
\hline \multicolumn{3}{|l|}{ EXPOSICIÓN AL RIESGO LABORAL } \\
\hline SI & 42 & 73,68 \\
\hline NO & 15 & 26,32 \\
\hline \multicolumn{3}{|c|}{ Riesgo Biológico (¿Cómo estuvo expuesto?) } \\
\hline Manipulando desechos & 3 & $5.26 \%$ \\
\hline Salpicadura de fluidos & 5 & $8.77 \%$ \\
\hline Al momento de retirar el catéter & 1 & $1.75 \%$ \\
\hline Al realizar venopunciones & 6 & $10.53 \%$ \\
\hline Descartando hojas de bisturí & 1 & $1.75 \%$ \\
\hline Desechando la aguja en un recipiente & 26 & $45.62 \%$ \\
\hline \multicolumn{3}{|l|}{ Riesgo Químico } \\
\hline Siempre <28-35] & 4 & 7,02 \\
\hline A menudo <21-28] & 17 & 29,82 \\
\hline Algunas Veces <14-21] & 27 & 47,37 \\
\hline Casi Nunca <7-14] & 6 & 10,53 \\
\hline Nunca [1-7] & 2 & 3,51 \\
\hline \multicolumn{3}{|l|}{ Riesgo Físico } \\
\hline Siempre <16-20] & 7 & $12.28 \%$ \\
\hline A menudo <12-16] & 27 & $47.37 \%$ \\
\hline Algunas Veces <8-12] & 20 & $35.09 \%$ \\
\hline Casi Nunca <4-8] & 2 & $3.51 \%$ \\
\hline Nunca [1-4] & 0 & $0.00 \%$ \\
\hline
\end{tabular}

\section{DISCUSION}

La bioseguridad es una doctrina de comportamiento encaminada a lograr actitudes y conductas que disminuyan el riesgo de adquirir enfermedades infectocontagiosas y transmisibles en los trabajadores de salud. El control de ello es eficaz solo si todas las personas que trabajan en el sector salud conocen las medidas de bioseguridad y la importancia de su uso en la atención de los pacientes, por ello se evaluó la relación entre medidas de Bioseguridad que aplican los profesionales de enfermería con la Exposición al riesgo laboral en el Hospital Santa María del Socorro, año 20132014, Ica.

De acuerdo con el estudio se señala que existen medidas de bioseguridad en Barreras Físicas Siempre en un 33,33\%, A menudo $42,11 \%$, Algunas veces $19,30 \%$, Casi nunca $5,26 \%$ y Nunca $0 \%$. Sin embargo en Venezuela Téllez et al, en su investigación concluyo que las medidas de bioseguridad no son observadas por un porcentaje importante y no usan las medidas de bioseguridad en general, en particular lo más importante está referido a la no utilización de lentes protectores(4) y Becerra encontró que el $99,22 \%$ hace uso correcto de guantes al momento de preparar tratamiento, que $0 \%$ utiliza protección ocular, que un $68 \%$ utiliza correctamente el respirador, tan solo un
$20,32 \%$ utiliza botas desechables, solo el $39,84 \%$ usa gorro y el $0 \%$ se coloca ropa impermeable (1).

En lo que se refiere a Barreras Biológicas se observa que el profesional de enfermería del Hospital Santa María del Socorro (Ica), respecto a la Vacuna de Hepatitis B está protegido con las tres dosis el $7,02 \%$, con la Vacuna Toxoide tetánico con las tres dosis en un $7,02 \%$, con la vacuna Influenza el $73,68 \%$, con la vacuna SR un $52,63 \%$ y con la Vacuna BCG un $89,47 \%$ de la muestra, similarmente Lozada et al en su investigación en el Hospital "Dr. Raúl Leoni Otero"(Venezuela) en donde los resultados obtenidos, señalan que El 69,6 \% del personal de enfermería conoce las barreras biológicas. El $100 \%$ del personal de enfermería tiene cumplida la vacuna que inmuniza contra la Hepatitis B. El 45,7\% de los profesionales de enfermería cumple con el esquema de la vacuna que inmuniza contra la Hepatitis B (1era dosis, 2da dosis, 3era dosis más el refuerzo) y el $87,0 \%$ del personal de enfermería tiene cumplida la vacuna que inmuniza contra el Tétano (7).

En lo que concierne a Precauciones estándar respecto al Lavado de Manos Siempre un $97,74 \%$, A menudo un $5,26 \%$, Algunas veces $0.00 \%$ casi nunca $0.00 \%$ y nunca $0,00 \%$. Coincidente con Becerra en 
su investigación concluye que el 95,31\% del personal realiza el lavado de manos antes de cada procedimiento, un $97,66 \%$ los realiza después de cada procedimiento y un $89,06 \%$ aplica las técnicas adecuadas al momento de lavarse las manos (1). También se considera que existen medidas de precaución estándar respecto a la disponibilidad de desechos Siempre un $71,93 \%$, A menudo un $22,81 \%$, Algunas veces $5,26 \%$ casi nunca $0,00 \%$ y nunca $0,00 \%$. Mientras que Becerra concluye que el $100 \%$ del personal maneja el material punzocortante y separa adecuadamente los desechos sólidos del material punzocortante (1) y Tellez concluye que el profesional de enfermería no hace un buen manejo de los desechos (4).

En cuanto a la exposición al Riesgo Laboral: ¿Alguna vez ha tenido riesgo de sufrir un accidente laboral en su servicio? El cual consideran que SI un $73,68 \%$ y que NO un $26,32 \%$ y Según Miguez et al en su investigación concluyo que se evidencio en su mayoría que el personal encuestado afirma haber sufrido accidentes laborales en el centro quirúrgico de los que se destacan: salpicaduras y cortaduras (8).

Se observa también que el profesional de enfermería considera respecto a la exposición al Riesgo Biológico: ¿Cómo estuvo expuesto al riesgo biológico?, El cual consideran que manipulando desechos un 5,26\%, salpicadura de fluidos un $8,77 \%$, Al momento de retirar el Catéter un $1,75 \%$, al realizar venopunciones un $10,53 \%$, descartando hojas de bisturí un $1,75 \%$ y desechando la aguja en un recipiente $45,62 \%$ mientras tanto Tellez menciona que existe una importante incidencia de accidentes laborales la mayoría por punciones percutáneas y cortaduras (4).

Singularmente a la exposición por Riesgo Físico es Siempre un 12,28\%, A menudo un $47,37 \%$, Algunas veces $35,09 \%$, Casi nunca un $3,51 \%$ y nunca un $0,00 \%$. Observándose en el estudio de Miguez que en su mayoría, se considera que los riesgos a los que se encuentra más expuesto el personal en el área del centro quirúrgico son los agentes físicos, también la mayoría del personal encuestado afirma no haber sido capacitado sobre las medidas a tomar en un accidente laboral (8).

\section{CONCLUSIONES}

Así mismo como el rho de Spearman es 0,244 afirmamos que Existe relación inversa baja pero no significativa entre las medidas de Bioseguridad de las Barreras de Protección que aplica el Profesional de Enfermería y la Exposición al Riesgo biológico.

Además como el rho de Spearman es -0,062 afirmamos que Existe relación inversa mínima pero no significativa entre las medidas de bioseguridad de las Barreras de Protección que aplica el Profesional de Enfermería y la Exposición al Riesgo físico.

Existe relación directa Moderada Significativa entre las Medidas de Bioseguridad entre las barreras de protección que aplica el Profesional de Enfermería están relacionadas con la Exposición al Riesgo Químico con el rho de Spearman 0.442 .

\section{CORRESPONDENCIA}

\section{LIC. SORIA QUISPE CAROLINA FIORELLA CORREO ELECTRONICO:}

fivi_love@hotmail.com

\section{REFERENCIAS BIBLIOGRAFICAS}

1. Becerra N, Calojero E. Aplicación de las Normas de Bioseguridad de los Profesionales de Enfermería (internet). Universidad del Oriente. Tesis de Grado. Cumaná, Venezuela. 2010. (citado en mayo 2013) Disponible en: http://ri.biblioteca.udo.edu.ve/bitstream/ 123456789/2198/1/15\%20Tesis.\%20QY9 \%20B389.pdf

2. Moreno F, Godoy E. Riesgos Laborales un Nuevo Desafío para la Gerencia. Rv. Daena: International Journal of Good Conscience. 7(1) 38-56. Monterrey, México. 2012 (internet) (citado en Junio 2013); Disponible en : http://www.spenta mexico.org/v7-n1/7(1)38-56.pdf 
3. Núñez Z, Ramírez D. Características epidemiológicas de los accidentes laborales punzocortantes y de exposición mucocutánea en el personal asistencial de enfermería del hospital Alberto Sabogal Sologuren - Es salud. Rv. Científica de Enfermería RECIEN Vol 1(2). Lima, Perú 2009. (Internet). (citado en mayo 2013) Disponible en: http://www.cep.org.pe/ cicep/ revista/volumen1/cap\%2037-42.pdf

4. Téllez J, Tovar M. Medidas de Bioseguridad que aplica el Profesional de enfermería y la Accidentabilidad Laboral en la Unidad Quirúrgica, Hospital "Dr. José María Vargas en el Segundo Semestre de 2007 (internet). (citado en julio 2013). Disponible en:

http://www.monografias.com/trabajos-

pdf/accidentalidad-laboral-unidad-

quirurgica/accidentalidad-laboral-unidadquirurgica.pdf

5. Uribe C. Riesgo laboral asociado a factores psicosociales del personal asistencial y administrativo del hospital Regional de Ica 2008-2009 (Ica). [tesis] de Doctorado en salud pública. Universidad Nacional San Luis Gonzaga de Ica, Perú. 2012.

6. Suarez M. ManualdeBioseguridad del Hospital Nacional Hipólito Unanue. (internet). (citado en julio 2013). Disponible en: http://www.hnhu.gob.pe/CUERPO/ EPIDEMIOLOGIA/SALA\%20SITUACIONA L\%202013/

MANUAL\%20DE\%20BIOSEGURIDAD\%2 OHNHU\%202013\%20Rev.pdf
7. Lozada M, Rodríguez G, Tovar $\mathrm{Y}$. Medidas para la prevención de riesgos biológicos que aplica el personal de Enfermería que labora en la unidad de emergencia del Hospital "Dr. Raúl Leoni Otero", San Félix, Estado Bolívar, primer trimestre 2.009. Universidad Central de Venezuela, Facultad de Medicina. Escuela de Enfermería. (Internet). (citado en Agosto 2013) Disponible en: http://saber.ucv.ve/xmlui/bitstream/123456 789/1147/1/TESIS\%20ESPECIAL\%20DE \%20GRADO\%20DE\%20MERIDA.pdf

8. Miguez H, Yanez X, Satos D. Riesgo Laboral del Personal de Enfermería relacionado con la Bioseguridad en el área de quirófano del Hospital Alfredo Noboa Montenegro Cantón Guaranda provincia Bolívar, durante el periodo de octubre del 2010 a marzo del 2011. Tesis de Grado. Universidad Estatal de Bolívar. Facultad de Ciencias de la Salud. Escuela de enfermería. 2011. Guaranda, Ecuador. (Internet). (citado en setiembre 2013) Disponible en: http://www.biblioteca.ueb. edu.ec/bitstream/15001/423/2/TEXTO\%20 RIESGO\%20LABORAL.pdf

Recibido: 10/03/14

Aprobado para publicación: 10/06/14 